\title{
APPROXIMATING POWER BY WEIGHTS
}

\author{
SASCHA KURZ
}

\begin{abstract}
Determining the power distribution of the members of a shareholder meeting or a legislative committee is a well-known problem for many applications. In some cases it turns out that power is nearly proportional to relative voting weights, which is very beneficial for both theoretical considerations and practical computations with many members. We present quantitative approximation results with precise error bounds for several power indices as well as impossibility results for such approximations between power and weights.
\end{abstract}

\section{INTRODUCTION}

Consider a stock corporation whose shares are owned by three major stockholders owning $35 \%, 34 \%$, and $17 \%$, respectively. The remaining $14 \%$ are widely spread. Assuming that decisions a made by simple majority rule, all three major stockholders have equal influence on the company's decisions, while the private shareholders have no say. To be more precise, any two major stockholders can adopt a proposal, while the private shareholders together with an arbitrary major stockholder need further affirmation. Such decision environments can be captured by means of weighted voting games. Formally, a weighted (voting) game consists of a set of players $N=\{1, \ldots, n\}$, a vector of non-negative weights $w=\left(w_{1}, \ldots, w_{n}\right)$, and a positive quota $q$. A proposal is accepted if and only if the weight sum of its supporters meets or exceeds the quota. Committees that decide between two alternatives have received wide attention. Von Neumann and Morgenstern introduced the notion of simple games, which is a super class of weighted games, in [39]. Examples of decision-making bodies that can be modeled as weighted games are the US Electoral College, the Council of the European Union, the UN Security Council, the International Monetary Fund or the Governing Council of the European Central Bank. Many applications seek to evaluate players' influence or power in simple or weighted games, see, e.g., [28]. The initial example illustrates that shares or weights can be a relative poor proxy for the distribution of power. Using the taxicab metric, i.e., the $\|\cdot\|_{1}$-distance, the corresponding distance between shares and relative power is $\left|0.35-\frac{1}{3}\right|+\left|0.34-\frac{1}{3}\right|+\left|0.17-\frac{1}{3}\right|+|0.14-0| \approx 32.67 \%$.

If the weights add up to one, then we speak of relative weights. The insight that the power distribution differs from relative weights, triggered the invention of so-called power indices like the Shapley-Shubik index [38], the Penrose-Banzhaf index [3], or the nucleolus [37]. Due to the combinatorial nature of most of those indices, qualitative assessments are technically demanding and large numbers of involved parties cause 
computational challenges [4]. Moreover, there is a large variety of different power indices proposed so far. On the positive side, there are a few limit results, which state that, under certain technical conditions, the power distribution of an infinite sequence of weighted voting systems tends to the relative weight distribution. This of course simplifies the analysis. The aim of this paper is to provide quantitative results of the form

$$
\|p-w\|_{1}=\sum_{i=1}^{n}\left|p_{i}-w_{i}\right| \leq \frac{c \Delta}{\min \{q, 1-q\}},
$$

where $w$ is the relative weight vector, $q$ the quota, $p$ the power distribution induced by a certain power index, $\Delta=\max _{i} w_{i}$ the maximum relative weight, and $c \in \mathbb{R}_{>0}$ a constant depending on the chosen power index. This inequality provides a concrete error bound based on just a few invariants of the underlying weighted game. Although limit results for sequences of weighted games can be derived in general, Inequality (1) can also be applied to a single weighted game. Applications range from approximating power distributions with many involved parties, where the exact evaluation is computationally infeasible, to statements about power distributions in situations with incomplete or uncertain information. In our above example there may be many private shareholders whose exact shares are either unknown (due to the lack of a reporting obligation) or highly volatile. (Our precise statement about the exact power distribution, independent of the distribution of the widely spread shareholdings and almost independent of the chosen power index, is due to a rather special situation, see the end of Subsection 2.1.) Results for different distance measures can be derived in a unified way, which makes the choice of the $\|\cdot\|_{1}$-distance less special. The precise involvement of the invariants $\Delta, q$, and $1-q$ in the right hand side of Inequality (1) is rather an explanation of a universal behavior than a limitation. We will derive lower bounds for the constant $c$ independent of the properties of the chosen power index, i.e., besides the constant, results like Inequality (1) are the best we can hope for. This explains the necessity of several conditions used in known limit results.

E.g., for the Shapley-Shubik index Neyman obtained in 1982:

Theorem 1.1. [34] Let $n \in \mathbb{N}, N=\{1, \ldots, n\}, 0<q<1, w \in \mathbb{R}_{>0}^{n}$ with $\|w\|_{1}=1$, and $P(i, q)$ be the probability that in a random order of $N, i$ is the first element in the order for which the $w$-accumulated sum exceeds $q$. For every $\varepsilon>0$ there exist constants $\delta>0$ and $K>0$ such that if $\rho=\max _{i \in N} w_{i}<\delta$, and $K \rho<q<1-K \rho$ then $\|P(\cdot, q)-w\|_{1}<\varepsilon$.

In other words, if the maximum relative weight $\Delta$ approaches zero and the relative quota $q$ is not too near to the extreme points 0 or 1 , then the power distribution tends to the vector of relative weights if the distance is measured by the $\|\cdot\|_{1}$-norm. The precise dependence of $\delta$ and $K$ on $\varepsilon$ is hidden in the technical lemmas of [34]. This is due to the fact that the original motivation behind this result was the study of the asymptotic value of non-atomic games. 
Another well-known limit theorem is the Penrose limit theorem (PLT). It is an unproven statement implicitly contained in [36]. Loosely speaking it states that, for a quota of one half, the ratio between the Penrose-Banzhaf indices of any two voters converges to the ratio between their weights as the number of voters increases. In [32] the authors used Theorem 1.1 in order to derive a version of the PLT for the Shapley-Shubik index for so-called replicative $q$-chains, where finitely many types of voters get replicated with a strictly positive frequency. In Lemma 4.1 we deduce a general PLT-type result from Inequality (1), cf. [25, Proposition 1]. Using a local central limit theorem (for normalized sums of independent random variables) Lindner and Machover, see [32], also obtained a PLT for the Penrose-Banzhaf index for $q=\frac{1}{2} \sum_{i=1}^{n} w_{i}$ and divisibility conditions on the involved (non-normalized) integer weights.

Besides the Shapley-Shubik and the Penrose-Banzhaf index, further limit results have only been obtained for the nucleolus. In [25, Lemma 1] the authors have proven Inequality (1) for $c=2$, which implies a PLT-type result.

One important problem in the context of power indices and weighted games, is the so-called "inverse power index problem", see e.g. [7, 19, 23, 24] and the references therein. It asks for weights and a quota such that the corresponding power distribution meets a given ideal power distribution as closely as possible. Since there is only a finite number of different weighted or simple games, it is obvious that some power vectors can not be approximated too closely if the number of voters is small. In [1] Alon and Edelman showed that there are also vectors that are hard to approximate by the PenroseBanzhaf index of a simple game if most of the mass of the vector is concentrated on a small number of coordinates. This goes in line with a relatively large maximum weight $\Delta$. Generalizations and impossibility results for other power indices have been obtained in [22].

The case the power distribution coincidentally matching relative weights has received special attention in the literature. For the Penrose-Banzhaf index, the subclass of spherically separable simple games has this property, see [14]. In [35] Peleg shows that a homogeneous constant-sum weighted game has a nucleolus which equals the relative homogeneous weights. A similar result for the nucleolus of weighted games with many replicated voters that have integral weights from a finite set has been obtained in [25, Proposition 2].

For more practically orientated studies on the ownership and control structure of stock companies we refer the interested reader, e.g., to [31]. Algorithms to approximate power indices can, e.g., be found in [2, 9, 29]. The expected difference between power and weights has been studied in [15] for the Shapley-Shubik and the Penrose-Banzhaf index. Intervals for the power of voters in weighted games with uncertain weights can also be computed with integer linear programming techniques, see [21]. However, these techniques (currently) are computationally infeasible for more than, say, 20 voters.

The rest of the paper is organized as follows. In Section 2 we introduce weighted games, power indices, norms and limits. Our main results are concluded in Section 3 . which is subdivided into three subsections. First we discuss invariants of weighted 
games that are suitable for upper bounds on the deviation of power and relative weights. In Lemma 3.14 we show that bounds of the form of Inequality (1) are the best that we can hope for. One such upper bound, which is applicable for the nucleolus, is obtained in Lemma 3.8. In combination with Lemma 3.12 all power indices based on weighted representations can be captured. We close this section by numerical investigations for other power indices. In Section 4 we briefly show how quotient-like statements as PLTtype results can be obtained in general from those $\|\cdot\|_{1}$ upper bounds. We close with a conclusion and future research directions in Section 5. The more technical proofs from results of Section 3 are evacuated to Section $\mathrm{A}$ in the appendix.

\section{Preliminaries}

This section collects some notation and basic facts. Simple games, weighted games and power indices as a tool for the measurement of voting power are treated in Subsection 2.1. Readers being familiar with the basics of cooperative game theory can safely skip this part. Subsection 2.2 treats the mathematical basics of distance functions, norms, and limits. Since some papers on limit results for power indices are rather imprecise in their statements, it is useful to briefly summarize the precise formalization and notation.

2.1. Weighted games, simple games and measurement of power. For a positive integer $n$ let $N=\{1, \ldots, n\}$ be the set of voters. A simple game is a mapping $v: 2^{N} \rightarrow\{0,1\}$ from the subsets of $N$ to binary outcomes satisfying $v(\emptyset)=0$, $v(N)=1$, and $v(S) \leq v(T)$ for all $\emptyset \subseteq S \subseteq T \subseteq N$. The interpretation in the context of binary voting systems is as follows. A subset $S \subseteq N$ is considered as the set of voters that are in favor of a proposal, i.e., which vote "yes". $v(S)$ encodes the group decision, i.e., $v(S)=1$ if the proposal is accepted and $v(S)=0$ otherwise. So, these assumptions are quite natural for a voting system with binary options in the input and output domain. A simple game $v$ is called weighted if there exist weights $w \in \mathbb{R}_{\geq 0}^{n}$ and a quota $q \in \mathbb{R}_{>0}$ such that $v(S)=1$ if and only if $\left.w(S):=\sum_{i \in S} w_{i} \geq q\right]^{1}$ From the conditions of a simple game we conclude $0<q \leq w(N)$. If $w(N)=1$ we speak of normalized or relative weights, where $0<q \leq 1$. We denote the respective game by $v=[q ; w]$ and refer to the pair $(q ; w)$ as a weighted representation, i.e., we can have $[q ; w]=\left[q^{\prime} ; w^{\prime}\right]$ but $(q ; w) \neq\left(q^{\prime} ; w^{\prime}\right)$. The example from the introduction can, e.g., be represented by $(51 \% ; 35 \%, 34 \%, 17 \%, 14 \%),\left(\frac{1}{2} ; \frac{1}{3}, \frac{1}{3}, \frac{1}{3}, 0\right)$, or $(6 ; 4,3,3,1)$, where the fourth voter mimics the private shareholders. Two voters $i, j \in N$ are called equivalent if $v(S \cup\{i\})=v(S \cup\{j\})$ for all $S \subseteq N \backslash\{i, j\}$. If $v(\{i\})=1$, we call voter $i$ a passer and a null voter if $v(S \cup\{i\})=v(S)$ for all $S \subseteq N \backslash\{i\}$.

A power index $\varphi$ is a family of mappings from the set of simple or weighted games on $n$ voters into $\mathbb{R}^{n}$, where $\varphi_{i}(v)$ denotes the $i$ th component of $\varphi(v) \in \mathbb{R}^{n}$. We call $\varphi$ positive if $\varphi(v) \in \mathbb{R}_{\geq 0}^{n} \backslash\{0\}$ for all possible games $v$. We say that $\varphi$ satisfies the null voter property if $\varphi_{i}(v)=0$ for each null voter $i \in N$. We call $\varphi$ symmetric if $\varphi_{i}(v)=$

\footnotetext{
${ }^{1}$ Some authors require $w(S)>q$, which may be written as $w(S) \geq q^{\prime}$ for $q^{\prime}$ slightly larger than $q$.
} 
$\varphi_{j}(v)$ for all equivalent voters $i, j$ and efficient if $\|\varphi(v)\|_{1}=1$ for all possible games $v$. However, if $\varphi$ is not efficient but positive, then $\varphi_{i}^{\prime}(v):=\varphi_{i}(v) / \sum_{j=1}^{n} \varphi_{j}(v)$ is both. The absolute Penrose-Banzhaf index is defined by $\frac{1}{2^{n-1}} \sum_{S \subseteq N \backslash\{i\}}(v(S \cup\{i\})-v(S))$ for voter $i \in N$. With this, the (relative) Penrose-Banzhaf index is the corresponding efficient version as constructed before. The Shapley-Shubik index for voter $i$ is given by $\sum_{S \subseteq N \backslash\{i\}} \frac{|S| ! \cdot(n-|S|-1) !}{n !} \cdot(v(S \cup\{i\})-v(S))$. In order to define the nucleolus of a simple game we need some preparations. In our context, an imputation $x$ is an element of $\mathbb{R}_{\geq 0}^{n}$ with $\|x\|_{1} \leq 1$. For an imputation $x$ and $S \subseteq N$ we call $e(S, x)=v(S)-$ $x(S)$ the excess, where $x(S)=\sum_{i \in S} x_{i}$. With this, the excess vector is the weakly monotonically decreasing list of the excesses of the $2^{n}$ subsets of $N$. E.g., for $v=$ $[4 ; 3,2,1,1]$ and $x=\left(\frac{1}{3}, \frac{1}{3}, \frac{1}{6}, \frac{1}{3}\right)$ the excess vector is given by

$$
\left(\frac{1}{2}, \frac{1}{2}, \frac{1}{3}, \frac{1}{3}, \frac{1}{3}, \frac{1}{6}, \frac{1}{6}, 0,0,-\frac{1}{6},-\frac{1}{6},-\frac{1}{3},-\frac{1}{3},-\frac{1}{3},-\frac{1}{2},-\frac{1}{2}\right) .
$$

The (unique) imputation $x^{\star}$ that yields the lexicographical minimal excess vector is called the nucleolus of $v$. See [37] for the original definition which does not apply to simple games with more than one passer. $\left\|x^{\star}\right\|_{1}=1$ is automatically satisfied in the minimum. Note that some authors require $\|x\|_{1}=1$ for any imputation. We remark that all three mentioned power indices are positive, symmetric, efficient and satisfy the null voter property.

In order to capture the special structure underlying the example from the introduction, we have to introduce a unanimity game $u_{S}$ as follows: $u_{S}(T)=1$ if and only if $S \subseteq T$, where $\emptyset \neq S \subseteq N$. For each symmetric and efficient power index $\varphi$ satisfying the null voter property we have $\varphi_{i}\left(u_{S}\right)=1 /|S|$ for all $i \in S$ and $\varphi_{i}\left(u_{S}\right)=0$ otherwise.

2.2. Mathematical basics of limits, norms and distance functions. A distance function or metric is used to measure the distance between two elements $x, y$ of some (arbitrary) set $U$ :

Definition 2.1. A metric on a set $U$ is a function (called the distance function) $d: U \times$ $U \rightarrow \mathbb{R}$, where for all $x, y, z \in U$ the following conditions are satisfied:

(1) $d(x, y) \geq 0$ (non-negativity or separation axiom);

(2) $d(x, y)=0 \Leftrightarrow x=y$ (identity of indiscernibles);

(3) $d(x, y)=d(y, x)$ (symmetry);

(4) $d(x, z) \leq d(x, y)+d(y, z)$ (subadditivity or triangle inequality).

Given a connected graph $G=(V, E)$ a metric on the set $V$ of its vertices is e.g. given by the length, i.e., the number of edges, of a shortest path connecting both vertices. We remark that property (or axiom) (1) can be concluded from the other ones. For a metric we assume no structure of the set $U$, which in turn allows a vast diversity of different metrics in general. Given a metric $d$ on a set $U$ we can compare any two elements of $U$ according to their distance. For a sequence $\left(x_{n}\right)_{n \in \mathbb{N}}$, i.e., an infinite ordered list of 
elements $\left.x_{n} \in U\right]^{2}$ in $U$, we can formalize the idea of the $x_{n}$ tending to some ultimate $x \in U$ as follows:

Definition 2.2. Given a metric space $(U, d)$, i.e., a set $U$ and a metric $d$ on $U$, we say that $x \in U$ is the limit of a sequence $\left(x_{n}\right)_{n \in \mathbb{N}}$ (in $U$ ) if for all $\varepsilon \in \mathbb{R}_{>0}$ there exists an $N(\varepsilon) \geq 0$ such that for all integers $n \geq N(\varepsilon)$, we have $d\left(x_{n}, x\right)<\varepsilon$. If a sequence admits a limit, we say that the sequence is convergent.

We remark that each convergent sequence uniquely determines a limit. However, whether a sequence converges can depend on the used metric. For our example of the graph metric the only convergent sequences are (almost) constant sequences, where for some arbitrary but finite integer $N_{0}, x_{n}=x$ for all $n \geq N_{0}$. The previous definition has the disadvantage that in order to decide whether the limit of a given sequence exists one needs to know or check all possible limits. Given a metric space $(U, d)$ a sequence $\left(x_{n}\right)_{n \in \mathbb{N}}$ (in $U$ ) is a Cauchy sequence if for all $\varepsilon \in \mathbb{R}_{>0}$ there exists an $N(\varepsilon) \geq 0$ such that for all integers $n, m \geq N(\varepsilon)$, we have $d\left(x_{n}, x_{m}\right)<\varepsilon$. A metric space $(U, d)$ in which every Cauchy sequence converges to an element of $U$ is called complete. In a complete metric space a sequence is convergent if and only if it is a Cauchy sequence.

A drawback of the above definition is that the convergence may depend on the precise definition of the used metric. We thus restrict attention to metrics induced by a norm of a finite dimensional vector space.

Definition 2.3. Given an $\mathbb{R}$-vector space $V$, a norm on $V$ is a function $\|\cdot\|: V \rightarrow \mathbb{R}$ with the following properties 3

(1) $\|\lambda u\|=|\lambda| \cdot\|u\|$ (absolute homogeneity or absolute scalability);

(2) $\|u+v\| \leq\|u\|+\|v\|$ (subadditivity or triangle inequality);

(3) if $\|u\|=0$ then $u$ is the zero vector (separates points)

for all $u, v \in V$ and $\lambda \in \mathbb{R}$.

Each norm $\|\cdot\|$ induces a distance function via $d(x, y):=\|x-y\|$. For $V=\mathbb{R}^{n}$ examples of norms are given by $\|x\|_{1}=\sum_{i=1}^{n}\left|x_{i}\right|$ and $\|x\|_{\infty}=\max _{1 \leq i \leq n}\left|x_{i}\right|$. Given a vector space $V$ two metrics $\|\cdot\|$ and $\|\cdot\|^{\prime}$ are called equivalent if there exist $l_{1}, l_{2}, u_{1}, u_{2} \in$ $\mathbb{R}_{>0}$ such that $l_{1}\|v\| \leq\|v\|^{\prime} \leq u_{1}\|v\|$ and $l_{2}\|v\|^{\prime} \leq\|v\| \leq u_{2}\|v\|^{\prime}$ for all $v \in V$. In a finite-dimensional vector space all norms are equivalent. As an example consider

$$
1 \cdot\|x\|_{\infty} \leq\|x\|_{1} \leq n \cdot\|x\|_{\infty} \quad \text { and } \quad \frac{1}{n} \cdot\|x\|_{1} \leq\|x\|_{\infty} \leq 1 \cdot\|x\|_{1}
$$

for all $n \in \mathbb{N}_{>0}$ and all $x \in \mathbb{R}^{n}=: V$. (Indeed the stated constants are tight as they are attained at $x=(1,0, \ldots, 0)$ and $x=(1, \ldots, 1)$.) So, in $\mathbb{R}^{n}$ a sequence is convergent with respect to a metric induced by norm $\|\cdot\|$ if and only if it is convergent with respect

\footnotetext{
${ }^{2}$ Readers interested in generalizations of sequences in general topological spaces may look at nets or Moore-Smith sequences.

${ }^{3}$ We remark that there is a more general definition of a norm for $\mathbb{F}$-vector spaces, where $\mathbb{F}$ is an arbitrary field and where the absolute value $|\cdot|$ can be replaced by some arbitrary norm for $\mathbb{F}$.
} 
to a metric induced by another norm $\|\cdot\|^{\prime}$, i.e., there is no need to explicitly state the used norm. (As long as the application does not call for a specific metric that is not induced by a norm.)

The bound $\|x\|_{\infty} \leq\|x\|_{1}$ can be slightly improved in our context.

Lemma 2.4. For $w, \bar{w} \in \mathbb{R}_{\geq 0}^{n}$ with $\|w\|_{1}=\|\bar{w}\|_{1}=1$, we have $\|w-\bar{w}\|_{\infty} \leq \frac{1}{2}\|w-\bar{w}\|_{1}$.

Proof. With $S:=\left\{1 \leq i \leq n \mid w_{i} \leq \bar{w}_{i}\right\}$ and $A:=\sum_{i \in S}\left(\bar{w}_{i}-w_{i}\right), B:=$ $\sum_{i \in N \backslash S}\left(w_{i}-\bar{w}_{i}\right)$, where $N=\{1, \ldots, n\}$, we have $A-B=0$ since $\|w\|_{1}=\|\bar{w}\|_{1}$ and $w, \bar{w} \in \mathbb{R}_{\geq 0}^{n}$. Thus, $\|w-\bar{w}\|_{1}=2 A$ and $\|w-\bar{w}\|_{\infty} \leq \max \{A, B\}=A$.

\section{INEQUALITIES BETWEEN WEIGHTS AND POWER INDICES}

We are interested in upper bounds for the distance between the relative weights $w$ of a weighted game $[q ; w]$ (with $n$ voters) and the corresponding power distribution $\varphi([q ; w])$. As argued in the previous subsection, we should limit our considerations on distance functions induced by a norm $\|\cdot\|$. While any two norms are equivalent for a fixed dimension $n$, the corresponding constants can of course depend on $n$. So, we have to explicitly state which norms we want to use. Here, we restrict ourselves onto the norms $\|\cdot\|_{1}$ and $\|\cdot\|_{\infty}$, which represent two kinds of extreme positions (in a certain sense) and have nice mathematical and algorithmic properties. Knowing $w,[q ; w], \varphi(\cdot)$ and $\|\cdot\|$ of course uniquely determines $\|w-\varphi([q ; w])\|$. We thus aim at deriving upper bounds only invoking few invariants of a given representation $(q ; w)$ and the corresponding weighted game $[q ; w]$. In Subsection 3.1 we briefly describe the invariants considered in this paper and discuss possible alternatives. The aim of Subsection 3.2 is to derive lower bounds for the distance between relative weights and power in the worst case. Upper bounds are treated in Subsection 3.3 .

3.1. Invariants of weighted games and their representations. We consider a weighted game with normalized representation $(q ; w)$, i.e., $w \in \mathbb{R}_{\geq 0}^{n}$ with $\|w\|_{1}=1$. Useful and easy invariants are the number of voters $n$, the quota $q \in(0,1]$, and the maximum weight $\max _{i} w_{i}=\|w\|_{\infty} \cdot{ }^{4}$ However, also more sophisticated invariants of weight vectors have been studied in applications. The so-called Laakso-Taagepera index a.k.a. Herfindahl-Hirschman index, c.f. [27], is used in Industrial Organization to measure the concentration of firms in a market, see, e.g., [6].

Definition 3.1. For $w \in \mathbb{R}_{\geq 0}^{n}$ with $w \neq 0$ the Laakso-Taagepera index is given by

$$
L(w)=\left(\sum_{i=1}^{n} w_{i}\right)^{2} / \sum_{i=1}^{n} w_{i}^{2} .
$$

\footnotetext{
${ }^{4}$ For an arbitrary representation $(q ; w)$ we consider the normalized quota $q / \sum_{i=1}^{n} w_{i}$ and the normalized relative weight $\max \left\{w_{i} / \sum_{j=1}^{n} w_{j} \mid 1 \leq i \leq n\right\}$.
} 
In general we have $1 \leq L(w) \leq n$. If the weight vector $w$ is normalized, then the formula simplifies to $\bar{L}(w)=1 / \sum_{i=1}^{n} w_{i}^{2}$. Under the name "effective number of parties" the index is widely used in political science to measure party fragmentation, see, e.g., [26]. We observe the following relations between the maximum relative weight $\Delta=\Delta(w)$ and the Laakso-Taagepera index $L(w)$ :

Lemma 3.2. For $w \in \mathbb{R}_{\geq 0}^{n}$ with $\|w\|_{1}=1$, we have

$$
\frac{1}{\Delta} \leq \frac{1}{\Delta(1-\alpha(1-\alpha) \Delta)} \leq L(w) \leq \frac{1}{\Delta^{2}+\frac{(1-\Delta)^{2}}{n-1}} \leq \frac{1}{\Delta^{2}}
$$

for $n \geq 2$, where $\alpha:=\frac{1}{\Delta}-\left\lfloor\frac{1}{\Delta}\right\rfloor \in[0,1)$. If $n=1$, then $\Delta=L(w)=1$.

PROOF. Optimize $\sum_{i=1}^{n} w_{i}^{2}$ with respect to the constraints $w \in \mathbb{R}^{n},\|w\|_{1}=1$, and $\Delta(w)=$ $\Delta$, see Section A for the technical details.

So, any lower or upper bound involving $L(w)$ can be replaced by a bound involving $\Delta$ instead. Since $\Delta$ has nicer analytical properties and requires less information on $w$, we stick to $\Delta$ in the following. We remark that there are similar inequalities for other indices measuring market concentration.

In the context of the study of limit theorems for power distributions of weighted games the Laakso-Taagepera index was suggested in [30]. However, the limit behavior of $L(w)$ is in one-to-one correspondence to the limit behavior of $1 / \Delta(w)$ :

Corollary 3.3. Let $\left(w^{m}\right)_{m \in \mathbb{N}}$ be a sequence of vectors with non-negative entries and $\left\|w^{m}\right\|_{1}=1$. (To be more precise, $w^{m} \in \mathbb{R}_{\geq 0}^{n_{m}}$ for some $n_{m} \in \mathbb{N}_{>0}$.) Then, we have

$$
\lim _{m \rightarrow \infty} \Delta\left(w^{m}\right)=0 \Longleftrightarrow \lim _{m \rightarrow \infty} L\left(w^{m}\right)=\infty .
$$

We leave the study of other possible invariants of weighted games and their representations for future research.

3.2. Lower bounds for the worst case approximation. In order to study the question which set of invariants permits a meaningful upper bound on the distance between relative weights and power, we consider constructions meeting the prescribed invariants to obtain lower bounds on the worst case approximation.

Since a large number of power indices is introduced in the literature and this stream does not seem to dry out, it would be very desirable to have approximation statements which hold for large classes of power indices. With almost no assumption on the power index we observe:

Lemma 3.4. Let $n \in \mathbb{N}_{>0}, q, \bar{q} \in(0,1], w, \bar{w} \in \mathbb{R}_{\geq 0}^{n}$ with $\|w\|_{1}=\|\bar{w}\|_{1}=1$, $\|\cdot\|$ be an arbitrary norm on $\mathbb{R}^{n}$ and $\varphi$ be a power index, then we have

$$
\max \{\|w-\varphi([q ; w])\|,\|\bar{w}-\varphi([\bar{q} ; \bar{w}])\|\} \geq \frac{\|w-\bar{w}\|}{2} .
$$


Proof. Using the triangle inequality yields $\|w-\varphi([q ; w])\|+\|\bar{w}-\varphi([\bar{q} ; \bar{w}])\| \geq$ $\|w-\bar{w}\|$ from which we can conclude the stated inequality.

So, instead of lower bounds for the distance between relative weights and power, we will consider lower bounds for the maximum distance between two relative weight vectors of the same weighted game being compatible with the considered invariants. The general lower bound of Lemma 3.4 will now be used to show that controlling the quota and the number of voters cannot yield reasonable limit results:

Lemma 3.5. For each $q \in(0,1]$ there exists a weighted game $v=[q ; w]=[q ; \bar{w}]$ with $n \geq 2$, where $w, \bar{w} \in \mathbb{R}_{\geq 0}^{n},\|w\|_{1}=\|\bar{w}\|_{1}=1,\|w-\bar{w}\|_{\infty} \geq \frac{1}{3}$, and $\|w-\bar{w}\|_{1} \geq \frac{2}{3}$.

PROOF. We give general constructions for different ranges of $q$ :

- $\frac{2}{3}<q \leq 1: w=\left(\frac{2}{3}, \frac{1}{3}, 0, \ldots, 0\right), \bar{w}=\left(\frac{1}{3}, \frac{2}{3}, 0, \ldots, 0\right)$;

- $\frac{1}{3}<q \leq \frac{2}{3}: w=\left(\frac{2}{3}, \frac{1}{3}, 0, \ldots, 0\right), \bar{w}=(1,0, \ldots, 0)$;

- $0<q \leq \frac{1}{3}: w=\left(\frac{2}{3}, \frac{1}{3}, 0, \ldots, 0\right), \bar{w}=\left(\frac{1}{3}, \frac{2}{3}, 0, \ldots, 0\right)$.

So, if we just know that the number of voters tends to infinity and the quotas are fixed to some arbitrary number in $(0,1]$ or some arbitrary subinterval of $(0,1]$, then no limit result is possible. For a single weighted game we can state a constant number as a lower bound for the distance between relative weights and power independent of the invariants $q$ and $n$, both in the distances induced by the $\|\cdot\|_{1}$ - and the $\|\cdot\|_{\infty}$-norm, respectively.

Similarly, it is not sufficient to require that the maximum relative weight $\Delta$ tends to zero, i.e., $L(w) \rightarrow \infty$, which would imply that the number of voters grows without bounds. In terms of a single weighted game, we construct a weighted representation consisting of any number of voters that is sufficiently large and exactly meets the chosen value of $\Delta$. Then we construct another weighted representation of the same weighted game whose distance to the first weight vector is lower bounded by a constant in the distance induced by the $\|\cdot\|_{1}$-norm.

Lemma 3.6. For each $\Delta \in(0,1)$ there exists a weighted game $v=[q ; w]=[q ; \bar{w}]$ with $n \geq \frac{4}{3 \Delta}+6$ voters, where $q \in(0,1), w, \bar{w} \in \mathbb{R}_{\geq 0}^{n}, \Delta(w)=\Delta(\bar{w})=\Delta,\|w\|_{1}=$ $\|\bar{w}\|_{1}=1,\|w-\bar{w}\|_{1} \geq \frac{2}{3}$, and $\|w-\bar{w}\|_{\infty} \geq \Delta / 2$.

PROOF. If $\Delta \geq \frac{2}{3}$, we can choose $q=1-\Delta, w=(\Delta, 1-\Delta, 0, \ldots, 0), \bar{w}=(1-$ $\Delta, \Delta, 0, \ldots, 0)$, so that $\|w-\bar{w}\|_{1}=2 \cdot(2 \Delta-1) \geq \frac{2}{3}$ and $\|w-\bar{w}\|_{\infty}=2 \Delta-1 \geq \Delta / 2$. If $0<\Delta<\frac{2}{3}$, we set $q=\Delta / 2, w_{2 i-1}=\bar{w}_{2 i}=\Delta, w_{2 i}=\bar{w}_{2 i-1}=\Delta / 2$ for $1 \leq i \leq a$, $w_{2 a+1}=w_{2 a+2}=w_{2 a+3}=\bar{w}_{2 a+4}=\bar{w}_{2 a+5}=\bar{w}_{2 a+6}=\frac{1}{3}-\frac{a \Delta}{2} \geq 0, w_{2 a+4}=w_{2 a+5}=$ $w_{2 a+6}=\bar{w}_{2 a+1}=\bar{w}_{2 a+2}=\bar{w}_{2 a+3}=0$, and $w_{i}=\bar{w}_{i}$ for all $2 a+7 \leq i \leq n$, where $a:=\left\lfloor\frac{2}{3 \Delta}\right\rfloor \geq 1$. With this, we have $\|w-\bar{w}\|_{1}=a \Delta+1-\frac{3 a \Delta}{2}=1-\frac{a \Delta}{2} \geq \frac{2}{3}$ and $\|w-\bar{w}\|_{\infty}=\Delta / 2$.

We remark that the excluded case $\Delta=1$ can be treated separately: For $\Delta=1=q$ we have $[1 ; 1,0, \ldots, 0]=[1 ; 0,1,0, \ldots, 0]$, where the two weight vectors have a $\|\cdot\|_{1^{-}}$ distance of 2 . For each $w, \bar{w} \in \mathbb{R}^{n}$ with $\Delta(w)=\Delta(\bar{w})$, we obviously have $\|w-\bar{w}\|_{\infty} \leq$ 
$\Delta(w)$. So, a constant lower bound for the $\mid \cdot \|_{\infty}$-norm can only exist if we slightly weaken the assumptions.

Lemma 3.7. For $\Delta \in(0,1]$ there exists a weighted game $v=[q ; w]=[\bar{q} ; \bar{w}]$ with $n \geq$ $\frac{1}{\Delta}+1 \geq 2, q, \bar{q} \in(0,1), w, \bar{w} \in \mathbb{R}_{\geq 0}^{n}, \Delta(w)=\Delta,\|w\|_{1}=\|\bar{w}\|_{1}=1,\|w-\bar{w}\|_{\infty} \geq \frac{1}{3}$.

PROOF. If $1>\Delta \geq \frac{2}{3}$ we can apply Lemma 3.6, so that we assume $\Delta<\frac{2}{3}$ in the following. For $a:=\lfloor 1 / \Delta\rfloor \geq 2$ we set $w_{i}=\Delta$ for $1 \leq i \leq a, \Delta>w_{a+1}=1-a \Delta \geq 0$, and $w_{i}=0$ for $a+2 \leq i \leq n$. If $w_{a+1}>0$, we set $q=w_{a+1}, \bar{w}_{1}=1-\varepsilon, \bar{w}_{i}=\varepsilon / a$ for $2 \leq i \leq a+1, \bar{w}_{i}=0$ for $a+2 \leq i \leq n$, and $\bar{q}=\varepsilon$, where $\varepsilon=\min \{2 / 3-\Delta, \Delta / 2\}$. If $w_{a+1}=0$, we set $q=\Delta, \bar{w}_{1}=1-\varepsilon, \bar{w}_{i}=\varepsilon /(a-1)$ for $2 \leq i \leq a$, and $\bar{w}_{i}=0$ for $a+1 \leq i \leq n$, and $\bar{q}=\varepsilon$, where $\varepsilon=\min \{2 / 3-\Delta, \Delta / 2\}$. With this, we have $\|w-\bar{w}\|_{\infty}=1-\Delta-\varepsilon \geq \frac{1}{3}$ in both cases.

So, we have shown that controlling either the relative quota or the maximum relative weight is not sufficient to obtain reasonable upper bounds for the distance between relative weights and power if the number of voters gets large. However, it is sufficient to control the quota $q$ and the maximum relative weight $\Delta$ for some power indices. (If $\Delta$ tends to zero, then the number of voters automatically tends to $\infty$ since $\Delta \geq \frac{1}{n}$. Due to Lemma 3.2 it would also be sufficient to control the quota and the Laakso-Taagepera index.)

3.3. Upper bounds for the distance between weights and power. We start with a rather general upper bound for all positive and efficient power indices $\varphi$ satisfying $\sum_{i \in S} \varphi_{i}([q ; w)] \geq q$ for every winning coalition $S$. This directly implies an upper bound for the nucleolus.

Lemma 3.8. Let $w \in \mathbb{R}_{\geq 0}^{n}$ with $\|w\|_{1}=1$ for an integer $n \in \mathbb{N}_{>0}$ and $0<q<1$. For each $x \in \mathbb{R}_{\geq 0}^{n}$ with $\|x\|_{1}=1$ and $x(S)=\sum_{s \in S} x_{s} \geq q$ for every winning coalition $S$ of $[q ; w]$, we have $\|w-x\|_{1} \leq \frac{2 \Delta}{\min \{q+\Delta, 1-q\}} \leq \frac{2 \Delta}{\min \{q, 1-q\}}$, where $\Delta=\Delta(w)$.

Proof. Consider a winning coalition $T$ such that $x(T)$ is minimal and invoke $x(T) \geq q$, see Section A for the technical details.

Corollary 3.9. Let $w \in \mathbb{R}_{\geq 0}^{n}$ with $\|w\|_{1}=1$ for an integer $n \in \mathbb{N}_{>0}$ and $0<q<1$. For each element $x$ of the nucleus 5 , which contains the nucleolus, of $[q ; w]$, we have $\|w-x\|_{1} \leq \frac{2 \Delta}{\min \{q+\Delta, 1-q\}} \leq \frac{2 \Delta}{\min \{q, 1-q\}}$, where $\Delta=\Delta(w)$.

Proof. We have $1-x(S) \leq E_{1}(x)$ for every winning coalition $S$ of $[q ; w]$, where $E_{1}(x)$ is the maximum excess. Since $1-w(S) \leq 1-q$ for every winning coalition $S$ of $[q ; w]$, we have $1-x(S) \leq 1-q$ as the maximum excess is minimized for all elements of the nucleus.

\footnotetext{
${ }^{5}$ The nucleus of a weighted game $[q ; w]$ is the set of all $x \in \mathbb{R}_{\geq 0}^{n}$ with $\|x\|_{1}=1$ that minimize the maximum excess $E_{1}(x)=\max _{S \subseteq N} v(S)-x(S)$. If $[q ; w]$ contains passers, then those $x$ may not be individually rational, i.e., $x_{i} \geq v(\{\bar{i}\})$ is violated. This case is excluded by some authors.
} 
Lemma 3.8 and Corollary 3.9 slightly tighten and generalize [25, Lemma 1]. From Lemma 2.4 we can directly conclude similar bounds for the $\|\cdot\|_{\infty}$-distance.

Some power indices $\varphi$ have the property that $\varphi([q ; w])$ is a feasible weight vector for a suitable quota $q^{\prime}$, i.e., $[q ; w]=\left[q^{\prime} ; \varphi([q ; w])\right]$. Examples are the minimum sum representation index, see [10], or power indices based on averaged representations from [16, 17]. For the Penrose-Banzhaf index, the subclass of spherically separable simple games has this property, see [14]. Thus, it is appealing to study upper bounds for the $\|\cdot\|_{1}$-distance between two relative weight vectors of the same weighted game. From Lemma 3.8 we can directly conclude the following two implications:

Corollary 3.10. Let $w, \bar{w} \in \mathbb{R}_{\geq 0}^{n}$ with $\|w\|_{1}=\|\bar{w}\|_{1}=1$ for an integer $n \in \mathbb{N}_{>0}$ and $0<q \leq \bar{q}<1$. If $[q ; w]=[\bar{q} ; \bar{w}]$ and $\Delta=\Delta(w)$, then we have $\|w-\bar{w}\|_{1} \leq \frac{2 \Delta}{\min \{q, 1-q\}}$.

Corollary 3.11. Let $w, \bar{w} \in \mathbb{R}_{>0}^{n}$ with $\|w\|_{1}=\|\bar{w}\|_{1}=1$ for an integer $n \in \mathbb{N}_{>0}$ and $0<q, \bar{q}<1$. If $[q ; w]=[\bar{q} ; \bar{w}]$, then we have

$\|w-\bar{w}\|_{1} \leq \max \left\{\frac{2 \Delta(w)}{\min \{q, 1-q\}}, \frac{2 \Delta(\bar{w})}{\min \{\bar{q}, 1-\bar{q}\}}\right\} \leq \frac{2 \Delta(w)}{\min \{q, 1-q\}}+\frac{2 \Delta(\bar{w})}{\min \{\bar{q}, 1-\bar{q}\}}$.

Unfortunately, those corollaries do not allow us to derive a bound on $\|w-\bar{w}\|_{1}$ which only depends on $q$ and $\Delta(w)$. However, we can obtain the following analog of Lemma 3.8 for losing instead of winning coalitions.

Lemma 3.12. Let $w \in \mathbb{R}_{\geq 0}^{n}$ with $\|w\|_{1}=1, \Delta=\Delta(w)$, and $0<q<1$. For each $x \in \mathbb{R}_{\geq 0}^{n}$ with $\|x\|_{1}=1$ and $x(S)=\sum_{s \in S} x_{s} \leq q$ for every losing coalition $S$ of $[q ; w]$, we have $\|w-x\|_{1} \leq \frac{4 \Delta}{\min \{q, 1-q\}}$. Moreover, if $q>\Delta(w)$, then $\|w-x\|_{1} \leq$ $\frac{2 \Delta}{\min \{q-\Delta, 1-q+\Delta\}} \leq \frac{2 \Delta}{\min \{q-\Delta, 1-q\}}$.

PROOF. Consider a losing coalition $T$ such that $x(T)$ is maximal and invoke $x(T) \leq q$. Section A provides technical details.

Intuitively, the inconspicuous condition $\Delta(w)<q$ is equivalent to the property that $[q ; w]$ does not contain passers.

Corollary 3.13. Let $w, \bar{w} \in \mathbb{R}_{\geq 0}^{n}$ with $\|w\|_{1}=\|\bar{w}\|_{1}=1, \Delta=\Delta(w)$, and $0<q, \bar{q}<1$. If $[q ; w]=[\bar{q} ; \bar{w}]$, then we have $\|w-\bar{w}\|_{1} \leq \frac{4 \Delta}{\min \{q, 1-q\}}$. Moreover, if additionally $[q ; w]$ does not contain any passer, then we have $\|w-\bar{w}\|_{1} \leq$

$$
\min \left\{\frac{2 \Delta}{\min \{q-\Delta, 1-q\}}, \frac{2 \Delta(\bar{w})}{\min \{\bar{q}-\Delta(\bar{w}), 1-\bar{q}\}}\right\} \leq \frac{2 \Delta}{\min \{q-\Delta, 1-q\}} .
$$

Proof. If $\bar{q} \geq q$, then $\bar{w}(S) \geq \bar{q} \geq q$ for every winning coalition $S$ of $[q ; w]$. Here, we can apply Lemma 3.8. Otherwise we have $\bar{w}(T)<\bar{q}<q$ for every losing coalition $T$ of $[q ; w]$ and Lemma 3.12 applies.

We remark that Lemma 3.8 and Lemma 3.12 are also valid for roughly weighted games, where coalitions with a weight sum being equal to $q$ may also be losing. So, one might ask the same question for $\alpha$-roughly weighted games, see [11, 13], where 
coalitions with weight sum below $q$ are losing and coalitions with weight sum above $\alpha q$ are winning.

From Corollary 3.13 we can deduce the following: If $w \in \mathbb{R}_{\geq 0}^{n}$ with $\|w\|_{1}=1$ and $0<q<1$ so that $[q ; w]$ does not contain any passer, then $\|w-\varphi([q ; w])\|_{1} \leq$ $\frac{2 \Delta(w)}{\min \{q-\Delta(w), 1-q\}}$ for $\varphi$ being the minimum sum representation index or one of the power indices based on averaged representations.

In the previous subsection we have argued that reasonable upper bounds on the distance between weights and power are impossible if only the relative quota or the maximum relative weight is taken into account. If both invariants are known, we have presented corresponding upper bounds for some power indices. So far we know that both invariants have to be involved in every upper bound somehow, but the tightest possible functional correlation is unknown. To that end, we provide:

Lemma 3.14. For each $w \in \mathbb{R}_{\geq 0}^{n}, 0<\hat{q}<1$ there exist $\bar{w} \in \mathbb{R}_{\geq 0}^{n}, 0<\bar{q}<1$ with $[\hat{q} ; w]=[\bar{q} ; \bar{w}]$ and $\|w-\bar{w}\|_{1} \geq c \cdot \min \left\{2, \frac{\Delta}{\min \{\hat{q}, 1-\hat{q}\}}\right\}$, where $\Delta=\Delta(w)$ and $c=\frac{1}{5}$.

PROOF. A construction of a matching representation $(\bar{q}, \bar{w})$ is provided in Section A. $\square$

So, using Lemma 3.4 and Lemma 3.14 , we see that Lemma 3.8 and Corollary 3.13 are tight up to the involved constant $c$.

\begin{tabular}{cccccccc}
\hline & Shapley- & Penrose- & & Public & Deegan & & Shift \\
$n$ & Shubik & Banzhaf & Johnston & Good & Packel & Shift & DP \\
\hline 3 & 0.33333 & 0.20000 & 0.50000 & 0.33333 & 0.00000 & 0.33333 & 0.00000 \\
4 & 0.50000 & 0.40000 & 0.75000 & 0.51429 & 0.30000 & 0.51429 & 0.50000 \\
5 & 0.60000 & 0.57895 & 0.87500 & 0.70330 & 0.50000 & 0.80769 & 0.75000 \\
6 & 0.66667 & 0.72222 & 1.00000 & 1.00000 & 0.71795 & 1.25763 & 1.24444 \\
7 & 0.71429 & 0.82609 & 1.13710 & 1.43590 & 1.16923 & 1.60131 & 1.55556 \\
8 & 0.75000 & 0.89552 & 1.29167 & 1.78649 & 1.49020 & 2.13108 & 2.08929 \\
9 & 0.77778 & 0.98154 & 1.49796 & 2.01504 & 1.71429 & 2.53762 & 2.43750 \\
\hline
\end{tabular}

TABLE 1. Necessary constant $c$ for the approximation of the normalized minimum sum integer representation.

In order to prove similar results for other power indices it suffices to consider an arbitrary weighted representation for each weighted game, since we can use Corollary 3.13 and the triangle inequality to transfer the result to any other weighted representation (while, of course, the involved constant of the upper bound has to be increased). We can use that insight also in the other direction, i.e., to numerically check whether such an upper bound for a given power index might exist at all. Table 1 lists the maximum necessary constant $c$ so that $\|\varphi([q ; w])-w\|_{1} \leq \frac{c \cdot \max _{i} w_{i}}{\min \{q, 1-q\}}$ for each weighted game with $n$ voters. As representation $(q ; w)$ we have chosen the normalization of a minimum sum 
integer representation, see e.g. [20]. There are 993061482 weighted games with $n=9$ voters, see [18]. The exact numbers are unknown for $n>9$. For the definitions of the considered power indices we refer, e.g., to [22].

For the Shapley-Shubik index the "worst case" examples can be easily guessed.

Lemma 3.15. For $n \geq 3, v=[n-1 ; n-1, \overbrace{1, \ldots, 1}^{n-1}], \bar{q}=\frac{1}{2}, \bar{w}=\left(\frac{1}{2}, \frac{1}{2 n-2}, \ldots, \frac{1}{2 n-2}\right)$ where $v=[\bar{q} ; \bar{w}]$ and $\|\bar{w}\|_{1}=1$, we have $\varphi(v)=\frac{1}{n(n-1)} \cdot\left((n-1)^{2}, 1, \ldots, 1\right)$ and $\|\varphi(v)-\bar{w}\|_{1}=\frac{n-2}{n}$ for the Shapley-Shubik index $\varphi$.

PROOF. For a voter $2 \leq i \leq n$ we only need to consider the winning coalition $S=$ $\{2, \ldots, n\}$, so that $\varphi_{i}(v)=\frac{(n-2) ! \cdot 1 !}{n !}=\frac{1}{n(n-1)}$ and $\varphi_{1}(v)=1-\sum_{i=2}^{n} \varphi_{i}(v)=\frac{n-1}{n}$.

We conjecture that Lemma 3.15 gives indeed the worst case scenario for the ShapleyShubik index. For the Penrose-Banzhaf index the very same example leads to the power distribution $\frac{1}{2^{n-1}+n-2} \cdot\left(2^{n-1}-1,1, \ldots, 1\right)$, so that the corresponding constant $c$ quickly tends to 1 . While this indeed gives the worst case example for $n \leq 8$, things get worse for larger $n$. To that end, let $w_{i}=2$ for $1 \leq i \leq m, w_{i}=1$ for $m+1 \leq i \leq 2 m$, and $q=\alpha \cdot 3 m$, where $m \geq 1$ and $\alpha \in(0,1)$. If $\bar{q}(m)$ and $\bar{w}(n)$ denote the normalized quota and weights, then the $\operatorname{limit}_{m \rightarrow \infty} \| \varphi\left([\bar{q}(m) ; \bar{w}(m)]-\bar{w}(m) \|_{1}\right.$ exists for the PenroseBanzhaf index $\varphi$. We have depicted the corresponding limits for different values of $\alpha$ in Figure 1 as dist, where $q=1000 \alpha$. We remark that the function is symmetric to $\alpha=\frac{1}{2}$ and takes values between zero and $\frac{1}{3}$. As a close approximation we have plotted the function $f(x)=\left|x-\frac{1}{2}\right|^{3} \cdot \frac{8}{3}$ labeled as cmp. So, the $\|\cdot\|_{1}$-distance between relative weights and the corresponding power distribution according to the PenroseBanzhaf index converges to a constant while the maximum relative weight $\Delta$ tends to zero for a fixed relative quota. There are only two types of voters with shares of $\frac{2}{3}$ and $\frac{1}{3}$, respectively. This example shows that it is not possible to derive a general PLTtype result for the Penrose-Banzhaf index if the relative quota does not equal $\frac{1}{2}$. In that direction numerical simulations and analytical results can be found in [5] and [33], respectively. For the other power indices from Table 1, besides the Shapley-Shubik index, similar deviations occur.

\section{IMPLICATIONS OF UPPER BOUNDS ON THE $\|\cdot\|_{1}$-DISTANCE}

If the $\|\cdot\|_{1}$-distance between power and weights is small and the relative weight sum of all voters sharing the same weight as voter $i$ does not vanish, then the quotient between power and relative weight for voter $i$ has to be close to 1 .

Lemma 4.1. Let $w \in \mathbb{R}_{\geq 0}^{n}$ with $\|w\|_{1}=1,0<q<1$ and $\varphi$ be a symmetric, efficient, and positive power index. If $\|\varphi([q ; w])-w\|_{1} \leq \varepsilon$, then

$$
1-\frac{\varepsilon}{2 \alpha} \leq \frac{\varphi_{i}([q ; w])}{w_{i}} \leq 1+\frac{\varepsilon}{2 \alpha}
$$

for all $1 \leq i \leq n$ with $w_{i}>0$, where $\alpha=w(S)>0$ and $S:=\left\{1 \leq j \leq n: w_{i}=w_{j}\right\}$. 


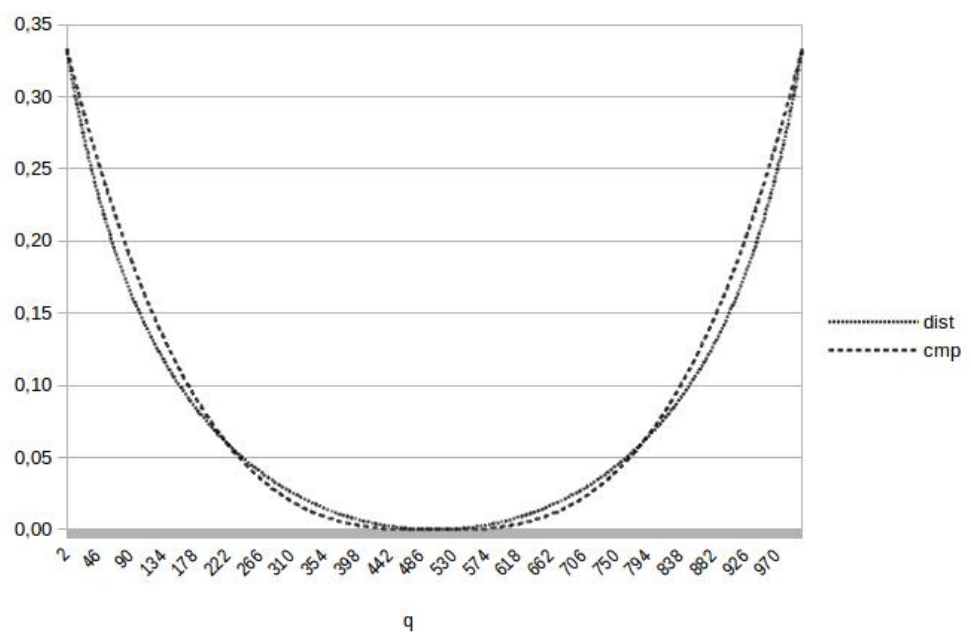

FIGURE 1. Deviation between weights and the Penrose-Banzhaf index.

Proof. Assuming $\frac{\varphi([q ; w])_{i}}{w_{i}}>1+\frac{\varepsilon}{2 \alpha}$ yields $\varphi(S)-w(S)>\varepsilon / 2$ by summing over all $j \in S$. Since $\|\varphi\|_{1}=\|w\|_{1}=1$ and $\varphi, w \in \mathbb{R}_{\geq 0}^{n}$ we would have $\|w-\varphi\|_{1}>\varepsilon-\mathrm{a}$ contradiction. Assuming $\frac{\varphi([q ; w])_{i}}{w_{i}}<1-\frac{\varepsilon}{2 \alpha}$ yields $w(S)-\varphi(S)>\varepsilon / 2$, which leads to the same contradiction.

Using the mass measure $\alpha$ is necessary since for each integer $n \geq 2$ we may consider the weighted game $v$ consisting of $n-1$ voters of weight 2 and one voter of weight 1 . Let $\varphi$ be a symmetric and efficient power index that satisfies the null voter property and $\bar{w}$ denote the corresponding relative weights. If the quota $q$ is an odd integer, we have $\varphi_{i}(v)=\frac{1}{n}$ for all $1 \leq i \leq n$, so that $\|\varphi(v)-\bar{w}\|_{1}=\frac{n-1}{n} \cdot \frac{2}{2 n-1}$. If $q$ is an even integer, then the voter with weight 1 is a null voter and all other voters get $\varphi_{i}(v)=\frac{1}{n-1}$ due to symmetry and efficiency. Here we have $\|\varphi(v)-\bar{w}\|_{1}=\frac{2}{2 n-1}$. So $\|\varphi(v)-\bar{w}\|_{1}$ tends to zero as the number $n$ of voters approaches infinity. However, the fraction $\frac{\varphi_{n}(v)}{\bar{w}_{i}}$ is either 0 or $2-\frac{1}{n}$, i.e., rather far away from 1 for larger $n$.

Bounds for quotients between power and weights for two involved players can be deduced from Lemma 4.1 via:

Lemma 4.2. If $w_{i}, w_{j}, \varphi_{i}, \varphi_{j} \in \mathbb{R}_{>0}, \varepsilon_{i}, \varepsilon_{j} \in[0,1)$ with $1-\varepsilon_{i} \leq \frac{\varphi_{i}}{w_{i}} \leq 1+\varepsilon_{i}$ and $1-\varepsilon_{j} \leq \frac{\varphi_{j}}{w_{j}} \leq 1+\varepsilon_{j}$, then

$$
\frac{1-\varepsilon_{i}}{1+\varepsilon_{j}} \leq \frac{w_{i}}{w_{j}} \cdot \frac{\varphi_{j}}{\varphi_{i}} \leq \frac{1+\varepsilon_{i}}{1-\varepsilon_{j}} \quad \text { and } \quad\left|\frac{\varphi_{i}}{w_{i}}-\frac{\varphi_{j}}{w_{j}}\right| \leq \varepsilon_{i}+\varepsilon_{j}
$$

\section{CONCLUSION}

If one is interested in upper bounds on the distance between relative weights and a corresponding power distribution or limits results for sequences of weighted games, 
given the relative quota and the maximum relative weight, Inequality (1) is the essential answer. In Section 4 we have shown that related results can be generally concluded.

We have derived upper bounds in the form of Inequality (1) for the nucleolus and all power indices based on weighted representations. Additionally, we have shown that for an arbitrary power index it is not possible to obtain bounds of smaller magnitude. So, future technical contributions might try to decrease the corresponding constants $c$ as far as possible (for the most important power indices). This contribution traded smaller constants for easier proofs. An important open problem is whether the Shapley-Shubik index permits an upper bound of the form of Inequality (1) or if the actual "convergence rate" is more slowly. While we showed that the monotonicity behavior of the LaaksoTaagepera index is the same as for the inverse maximum relative weight, it might still be possible that the Laakso-Taagepera index permits tighter bounds than the maximum relative weight. Moreover, it seems worthwhile to study other invariants than those used here.

Regarding limit results we showed that the power distribution tends to the relative weights under the $\|\cdot\|_{1}$-distance for the nucleolus and power indices based on weighted representations as long as the maximum relative weight tends to zero and the quotas are not too skewed (i.e., bounded away from 0 and 1.) An analytical example with two types of voters having non-vanishing weight shares illustrates that the $\|\cdot\|_{1}$-distance between the Penrose-Banzhaf power distribution and the relative weights tends to a strictly positive number provided that the quota is a fixed number different from $\frac{1}{2}$. So, for the Penrose-Banzhaf index power can not converge to weights provided the relative quota is not pegged at one half. Besides the Shapley-Shubik index, for which the general convergence is proven, there seems to be no suitable candidate for another such limit result among the classical power indices.

The case of the maximum relative weight going to zero is also sometimes called the non-atomic world. In contrast, in an atomic world some voters have a relatively large weight and all others have an individual weight that is comparatively negligible. However, this is not the case for the weight sum of all "small" voters. Limit results are available for the Shapley-Shubik and the Penrose-Banzhaf index in the atomic world, see [8]. Our example on the ownership structure of a stock corporation from the introduction very like belong to the atomic world. [30] claimed to unify limit results for the atomic and the non-atomic world. Lemma 3.2 and Corollary 3.3 show very transparently that this is not the case. Lemma 3.14 rather shows that it is impossible to derive meaningful bounds for those cases. However, it seems reasonable to assume that the weights of the large voters are known with high accuracy and that their number is relatively small. This would allow to make use of combinatorial algorithms. The idea is to solve an auxiliary problem to compute an approximation for the power distribution of the large voters. Suppose that for a set $N$ of voters we classify the voters into many small ones, collected in $O$, and a few large ones, collected in $N \backslash O$. Let $\bar{w}$ be the vector of relative weights, $\bar{q}$ be the relative quota, $\alpha=\bar{w}(O)$ the weight mass of the small 
voters, and $x$ be an optimal solution of

$$
\begin{aligned}
\min y & \text { subject to } \\
y+\sum_{i \in S} x_{i} \geq 1 & \forall S \subseteq N \backslash O: \bar{w}(S) \geq \bar{q} \\
y+\frac{\bar{q}-\bar{w}(S)}{\alpha} \cdot \beta+\sum_{i \in S} x_{i} \geq 1 & \forall S \subseteq N \backslash O: \bar{q}-\alpha \leq \bar{w}(S)<\bar{q} \\
\beta+\sum_{i \in N \backslash O} x_{i}=1 & \\
x_{i} \in \mathbb{R}_{\geq 0} & \forall i \in N \backslash O \\
\beta \in \mathbb{R}_{\geq 0} &
\end{aligned}
$$

We claim that $x_{i}^{\star}=x_{i}$ for $i \in N \backslash O$ and $x_{i}^{\star}=\overline{w_{i}} \cdot \frac{\beta}{\alpha}$ is a good approximation for the nucleolus $\bar{x}$ of $[\bar{q} ; \bar{w}]$. More precisely, we conjecture that there exists a constant $c \in \mathbb{R}_{>0}$ such that

$$
\left\|\bar{x}-x^{\star}\right\|_{1} \leq \frac{c \Delta_{O}}{\min \{|\bar{q}-\bar{w}(S)|: S \subseteq N \backslash O\}},
$$

where $\Delta_{O}=\min \left\{\bar{w}_{i}: i \in O\right\}$ is the maximum relative weight of a small voter. The idea is to treat the small voters as a continuum and to determine a vector that minimizes the maximum excess. This is the first step of the optimization problem for the nucleolus. Preliminary results in the direction of this conjecture were obtained in [12] quite some years ago. For suitable auxiliary problems for the Shapley-Shubik and the PenroseBanzhaf index we refer to [8]. For the latter the problem of corresponding limit results is widely open.

\section{ACKNOWLEDGMENT}

The author wishes to thank Alexander Mayer for his comments on an earlier draft of this paper.

\section{APPENDiX A. DELAYED PROOFS}

\section{ProOF. (Lemma 3.2)}

For $n=1$, we have $w_{1}=1, \Delta(w)=1, \alpha=0$, and $L(w)=1$, so that we assume $n \geq 2$ in the remaining part of the proof. For $w_{i} \geq w_{j}$ consider $a:=\frac{w_{i}+w_{j}}{2}$ and $x:=w_{i}-a$, so that $w_{i}=a+x$ and $w_{j}-x$. With this we have $w_{i}^{2}+w_{j}^{2}=2 a^{2}+2 x^{2}$ and $\left(w_{i}+y\right)^{2}+\left(w_{j}-y\right)^{2}=2 a^{2}+2(x+y)^{2}$. Let us assume that $w^{\star}$ minimizes $\sum_{i=1}^{n} w_{i}^{2}$ under the conditions $w \in \mathbb{R}_{\geq 0},\|w\|_{1}=1$, and $\Delta(w)=\Delta$. (Since the target function is continuous and the feasible set is compact and non-empty, a global minimum indeed exists.) W.1.o.g. we assume $w_{1}^{\star}=\Delta$. If there are indices $2 \leq i, j \leq n$ with $w_{i}^{\star}>w_{j}^{\star}$, i.e., $x>0$ in the above parameterization, then we may choose $y=-x$. Setting $w_{i}^{\prime}:=w_{i}^{\star}+y=a=\frac{w_{i}^{\star}+w_{j}^{\star}}{2}, w_{j}^{\prime}:=w_{j}^{\star}-y=a=\frac{w_{i}^{\star}+w_{j}^{\star}}{2}$, and $w_{h}^{\prime}:=w_{h}^{\star}$ 
for all $1 \leq h \leq n$ with $h \notin\{i, j\}$, we have $w^{\prime} \in \mathbb{R}_{\geq 0}^{n},\left\|w^{\prime}\right\|_{1}=1, \Delta\left(w^{\prime}\right)=\Delta$, and $\sum_{h=1}^{n}\left(w_{h}^{\prime}\right)^{2}=\sum_{h=1}^{n}\left(w_{h}^{\star}\right)^{2}-x^{2}$. Since this contradicts the minimality of $w^{\star}$, we have $w_{i}^{\star}=w_{j}^{\star}$ for all $2 \leq i, j \leq n$, so that we conclude $w_{i}^{\star}=\frac{1-\Delta}{n-1}$ for all $2 \leq i \leq n$ from $1=\left\|w^{\star}\right\|_{1}=\sum_{h=1}^{n} w_{h}^{\star}$. Thus, $L(w) \leq 1 /\left(\Delta^{2}+\frac{(1-\Delta)^{2}}{n-1}\right)$, which is tight. Since $\Delta \leq 1$ and $n \geq 2$, we have $1 /\left(\Delta^{2}+\frac{(1-\Delta)^{2}}{n-1}\right) \leq \frac{1}{\Delta^{2}}$, which is tight if and only if $\Delta=1$, i.e., $n-1$ of the weights have to be equal to zero.

Now, let us assume that $w$ maximizes $\sum_{i=1}^{n} w_{i}^{2}$ under the conditions $w \in \mathbb{R}_{\geq 0}$, $\|w\|_{1}=1$, and $\Delta(w)=\Delta$. (Due to the same reason a global maximum indeed exists.) Due to $1=\|w\|_{1} \leq n \Delta$ we have $0<\Delta \leq 1 / n$, where $\Delta=1 / n$ implies $w_{i}=\Delta$ for all $1 \leq i \leq n$. In that case we have $L(w)=n$ and $\alpha=0$, so that the stated lower bounds for $L(w)$ are valid. In the remaining cases we assume $\Delta>1 / n$. If there would exist two indices $1 \leq i, j \leq n$ with $w_{i} \geq w_{j}, w_{i}<\Delta$, and $w_{j}>0$, we may strictly increase the target function by moving weight from $w_{j}$ to $w_{i}$ (this corresponds to choosing $y>0$ ), by an amount small enough to still satisfy the constraints $w_{i} \leq \Delta$ and $w_{j} \geq 0$. Since $\Delta>0$, we can set $a:=\lfloor 1 / \Delta\rfloor \geq 0$ with $a \leq n-1$ due to $\Delta>1 / n$. Thus, for a maximum solution, we have exactly $a$ weights that are equal to $\Delta$, one weight that is equal to $1-a \Delta \geq 0$ (which may indeed be equal to zero), and $n-a-1$ weights that are equal to zero. With this and $a \Delta=1-\alpha \Delta$ we have $\sum_{i=1}^{n} w_{i}^{2}=a \Delta^{2}(1-a \Delta)^{2}=$ $\Delta-\alpha \Delta^{2}+\alpha^{2} \Delta^{2}=\Delta\left(1-\alpha \Delta+\alpha^{2} \Delta\right)=\Delta(1-\alpha(1-\alpha) \Delta) \leq \Delta$. Here, the latter inequality is tight if and only if $\alpha=0$, i.e., $1 / \Delta \in \mathbb{N}$.

\section{Proof. (Lemma 3.8)}

We set $N=\{1, \ldots, n\}, w(U)=\sum_{u \in U} w_{u}$ and $x(U)=\sum_{u \in U} x_{u}$ for each $U \subseteq N$. Let $S^{+}=\left\{i \in N \mid x_{i}>w_{i}\right\}$ and $S^{-}=\left\{i \in N \mid x_{i} \leq w_{i}\right\}$, i.e., $S^{+}$and $S^{-}$partition the set $N$ of players. We have $w\left(S^{+}\right)<1$ since $w\left(S^{+}\right)<x\left(S^{+}\right) \leq x(N)=1$, so that $w\left(S^{-}\right)>0$. Define $0 \leq \delta \leq 1$ by $x\left(S^{-}\right)=(1-\delta) w\left(S^{-}\right)$. We have

$$
x\left(S^{+}\right)=1-x\left(S^{-}\right)=w\left(S^{+}\right)+w\left(S^{-}\right)-(1-\delta) w\left(S^{-}\right)=w\left(S^{+}\right)+\delta w\left(S^{-}\right)
$$

and

$$
\|w-x\|_{1}=\left(x\left(S^{+}\right)-w\left(S^{+}\right)\right)+\left(w\left(S^{-}\right)-x\left(S^{-}\right)\right)=2 \delta w\left(S^{-}\right) .
$$

Generate a set $T$ by starting at $T=\emptyset$ and successively add a remaining player $i$ in $N \backslash T$ with minimal $x_{i} / w_{i}$, where all players $j$ with $w_{j}=0$ are the worst ones. Stop if $w(T) \geq q$. By construction $T$ is a winning coalition of $[q ; w]$ with $w(T)<q+\Delta$, since the generating process did not stop earlier and $w_{j} \leq \Delta(w)$ for all $j \in N$.

If $w\left(S^{-}\right) \geq q$, we have $T \subseteq S^{-}$and $x(T) / w(T) \leq x\left(S^{-}\right) / w\left(S^{-}\right)=1-\delta$. Multiplying by $w(T)$ and using $w(T)<q+\Delta$ yields

$$
x(T) \leq(1-\delta) w(T)<(1-\delta)(q+\Delta)=(1-\delta) q+(1-\delta) \Delta .
$$


Since $x(T) \geq q$, as $T$ is a winning coalition, we conclude $\delta<\Delta /(q+\Delta)$. Using this and $w\left(S^{-}\right)<1$ in Equation (3) yields

$$
\|w-x\|_{1}<\frac{2 \Delta}{q+\Delta}<\frac{2 \Delta}{q} .
$$

If $w\left(S^{-}\right)<q$, we have $S^{-} \subseteq T, x(T)=x\left(S^{-}\right)+x\left(T \backslash S^{-}\right), w\left(T \backslash S^{-}\right)>0$, and $w\left(S^{+}\right)>0$. Since $T \backslash S^{-} \subseteq S^{+}, x\left(T \backslash S^{-}\right) / w\left(T \backslash S^{-}\right) \leq x\left(S^{+}\right) / w\left(S^{+}\right)$, so that

$$
\begin{aligned}
x(T) & =x\left(S^{-}\right)+x\left(T \backslash S^{-}\right) \leq(1-\delta) w\left(S^{-}\right)+\frac{x\left(S^{+}\right)}{w\left(S^{+}\right)} \cdot\left(w(T)-w\left(S^{-}\right)\right) \\
& \leq(1-\delta) w\left(S^{-}\right)+\frac{x\left(S^{+}\right)}{w\left(S^{+}\right)} \cdot\left(q+\Delta-w\left(S^{-}\right)\right) \\
& =q+\frac{x\left(S^{+}\right) \Delta-(1-q) \delta w\left(S^{-}\right)}{w\left(S^{+}\right)} \\
& \leq q+\frac{\Delta-(1-q) \delta w\left(S^{-}\right)}{w\left(S^{+}\right)} .
\end{aligned}
$$

Since $x(T) \geq q$, we conclude $(1-q) \delta w\left(S^{-}\right) \leq \Delta$, so that $\|w-x\|_{1} \leq \frac{2 \Delta}{1-q}$.

\section{ProOF. (Lemma 3.12)}

If $q \leq 2 \Delta$, then $\frac{4 \Delta}{\min \{q, 1-q\}} \geq \frac{4 \Delta}{q} \geq 2 \geq\|x-w\|_{1}$, so that we can assume $q>\Delta$.

Using the notation from the proof of Lemma 3.8, we have $x\left(S^{+}\right)=w\left(S^{+}\right)+\delta w\left(S^{-}\right)$ and $\|w-x\|_{1}=2 \delta w\left(S^{-}\right)$.

Generate $T$ by starting at $T=\emptyset$ and successively add a remaining player $i$ in $N \backslash T$ with maximal $x_{i} / w_{i}$, where all players $j$ with $w_{j}=0$ are taken in the first rounds, as long as $w(T)+w_{i}<q$. By construction $T$ is a losing coalition of $[q ; w]$ with $q-\Delta \leq w(T)<q$, since the generating process did not stop earlier.

If $w\left(S^{+}\right) \geq q$, we have $T \subseteq S^{+}$and $x(T) / w(T) \geq x\left(S^{+}\right) / w\left(S^{+}\right)=1+\frac{\delta w\left(S^{-}\right)}{w\left(S^{+}\right)} \geq$ $1+\delta w\left(S^{-}\right)$. Multiplying by $w(T)$ and using $w(T) \geq q-\Delta$ yields

$$
x(T) \geq\left(1+\delta w\left(S^{-}\right)\right) w(T) \geq\left(1+\delta w\left(S^{-}\right)\right)(q-\Delta)=(q-\Delta)+\delta w\left(S^{-}\right)(q-\Delta) .
$$

Since $x(T) \leq q$, as $T$ is a losing coalition, we conclude $\delta w\left(S^{-}\right) \leq \Delta /(q-\Delta)$, so that $\|w-x\|_{1}<\frac{2 \Delta}{q-\Delta}$.

If $w\left(S^{+}\right)<q$, we have $S^{+} \subseteq T, x(T)=x\left(S^{+}\right)+x\left(T \backslash S^{+}\right), w\left(T \backslash S^{+}\right)>0$, and $w\left(S^{-}\right)>0$. Since $T \backslash S^{+} \subseteq S^{-}, x\left(T \backslash S^{+}\right) / w\left(T \backslash S^{+}\right) \geq x\left(S^{-}\right) / w\left(S^{-}\right)$, so that

$$
\begin{aligned}
x(T) & =x\left(S^{+}\right)+x\left(T \backslash S^{+}\right) \geq w\left(S^{+}\right)+\delta w\left(S^{-}\right)+\frac{x\left(S^{-}\right)}{w\left(S^{-}\right)} \cdot\left(w(T)-w\left(S^{+}\right)\right) \\
& \geq w\left(S^{+}\right)+\delta w\left(S^{-}\right)+(1-\delta) \cdot\left(q-\Delta-w\left(S^{+}\right)\right) \\
& =\delta w\left(S^{-}\right)+q-\Delta-\delta q+\delta \Delta+\delta w\left(S^{+}\right)=q-\Delta+\delta(1-q+\Delta) .
\end{aligned}
$$

Since $x(T) \leq q, \delta \leq \frac{\Delta}{1-q+\Delta}$, so that $\|w-x\|_{1} \leq \frac{2 \Delta}{1-q+\Delta}$ due to $w\left(S^{-}\right) \leq 1$. 
So, for $q>\Delta$ we have $\|w-x\|_{1} \leq \frac{2 \Delta}{\min \{q-\Delta, 1-q+\Delta\}} \leq \frac{2 \Delta}{\min \{q-\Delta, 1-q\}}$. In order to show $\|w-x\|_{1} \leq \frac{4 \Delta}{\min \{q, 1-q\}}$ it remains to consider the case $q \leq 1-q$. For $q>2 \Delta$, see the start of the proof, we have $\|w-x\|_{1} \leq \frac{2 \Delta}{\min \{q-\Delta, 1-q\}} \leq \frac{2 \Delta}{q-\Delta} \leq \frac{4 \Delta}{q} \leq \frac{4 \Delta}{\min \{q, 1-q\}}$.

In order to prove Lemma 3.14 , we need two preparing lemmas.

Lemma A.1. For each $0<q \leq \Delta \leq 1$, each $n \in \mathbb{N}$, and each $0<\varepsilon<\min \{\Delta, 1 / 2\}$ with $n \geq \max \{3,1 / \Delta\}$ there exist $w, \bar{w} \in \mathbb{R}_{\geq 0}^{n}$ with $\|w\|_{1}=\|\bar{w}\|_{1}=1, \Delta(w)=\Delta$, and $0<\bar{q}<1$ with $[q ; w]=[\bar{q} ; \bar{w}],\|w-\bar{w}\|_{1} \geq 1-2 \varepsilon$, and $\|w-\bar{w}\|_{\infty} \geq \frac{1}{2}-\varepsilon$.

Proof. We set $a:=\left\lfloor\frac{1}{\Delta}\right\rfloor \in \mathbb{N}_{\geq 1}$, so that $\frac{1}{2 \Delta}<a \leq \frac{1}{\Delta}$.

If $1-a \Delta \geq q$, we set $w_{i}=\Delta$ for $1 \leq i \leq a, w_{a+1}=1-a \Delta, w_{i}=0$ for $a+2 \leq i \leq n$, $\bar{w}_{a+1}=1-\varepsilon, \bar{w}_{i}=\varepsilon / a$ for $1 \leq i \leq a, \bar{w}_{i}=0$ for $a+2 \leq i \leq n$, and $\bar{q}=\varepsilon / a$. Here, we have $\|w-\bar{w}\|_{1}=2 \cdot(a \Delta-\varepsilon)>1-2 \varepsilon$ and $\|w-\bar{w}\|_{\infty}=a \Delta-\varepsilon \geq \frac{1}{2}-\varepsilon$.

If $1-a \Delta<q$ and $a \geq 2$, then we set $w_{i}=\Delta$ for $1 \leq i \leq a, w_{a+1}=1-a \Delta, w_{i}=0$ for $a+2 \leq i \leq n, \bar{w}_{1}=1-\varepsilon, \bar{w}_{i}=\frac{\varepsilon}{a-1}$ for $2 \leq i \leq \bar{a}, \bar{w}_{i}=0$ for $a+1 \leq i \leq n$, and $\bar{q}=\frac{\varepsilon}{a-1}$. With this, we have $\|w-\bar{w}\|_{1}=1-\varepsilon-\Delta+(a-1) \cdot\left(\Delta-\frac{\varepsilon}{a-1}\right)+1-a \Delta=$ $1+(a-2) \Delta-2 \varepsilon+(1-a \Delta) \geq 1-2 \varepsilon$ and $\|w-\bar{w}\|_{\infty} \geq 1-\Delta-\varepsilon \geq \frac{1}{2}-\varepsilon$ since $a \geq 2$, so that $\Delta \leq \frac{1}{2}$.

If $a=1$ and $1-a \Delta=1-\Delta<q$, then we have $\frac{1}{2}<\Delta \leq 1$ and we set $w_{1}=\Delta$, $w_{2}=1-\Delta, w_{i}=0$ for $3 \leq i \leq n, \bar{w}_{1}=\frac{1}{2}+\varepsilon, \bar{w}_{2}=0, \bar{w}_{3}=\frac{1}{2}-\varepsilon, \bar{w}_{i}=0$ for $4 \leq i \leq n$, and $\bar{q}=\frac{1}{2}$. With this, we have $\|w-\bar{w}\|_{1} \geq 1-2 \varepsilon$ and $\|w-\bar{w}\|_{\infty} \geq \frac{1}{2}-\varepsilon$.

Lemma A.2. Let $0<\varepsilon<\frac{1}{2}, 0<q<1, b \in \mathbb{N}_{\geq 1}, \frac{q}{b+1} \leq \Delta<\frac{q}{b}$, and $n \in \mathbb{N}$ with $n \geq \frac{1}{\Delta}+1$. Then, there exist $w, \bar{w} \in \mathbb{R}_{\geq 0}^{n}$ with $\|w\|_{1}=\|\bar{w}\|_{1}=1, \Delta(w)=\Delta$, and $0<\bar{q}<1$ with $[q ; w]=[\bar{q} ; \bar{w}],\|w-\bar{w}\|_{1}>\frac{2}{9} \cdot \frac{\Delta}{q}-\varepsilon$, and $\|w-\bar{w}\|_{\infty}>\frac{\Delta}{3(\Delta+1)} \cdot \frac{\Delta}{q}-2 \Delta \varepsilon$.

Proof. We set $a:=\left\lfloor\frac{1}{\Delta}\right\rfloor \in \mathbb{N}_{\geq 1}$, so that $\frac{1}{2 \Delta}<a \leq \frac{1}{\Delta}$. Consider $w_{i}=\Delta$ for $1 \leq i \leq a$, $0 \leq w_{a+1}=1-a \Delta<\Delta$, and $w_{i}=0$ for $a+2 \leq i \leq n$. Observe $\frac{1}{b}>\frac{\Delta}{q}$.

If $b \Delta+1-a \Delta<q$ we set $\kappa:=a$ and $\kappa:=a+1$ otherwise. So, the voters $\kappa+1 \leq i \leq n$ are null voters and the other voters are pairwise equivalent. We have $\kappa \geq 2$ since $\kappa=1$ implies $a=1$ and $\Delta>\frac{1}{2}$, so that $b=1$ and $b \Delta+1-a \Delta=1 \geq q$. Additionally, we have $\frac{\Delta}{\Delta+1} \leq \frac{1}{\kappa}<2 \Delta$. (The right hand side may be decreased to $\frac{3}{2} \Delta$.)

If $\kappa \equiv 0(\bmod 2)$, then we set $\bar{w}_{i}=\frac{2}{\kappa} \cdot \frac{b+1}{2 b+1}-\frac{\varepsilon}{\kappa}$ for $1 \leq i \leq \kappa / 2, \bar{w}_{i}=\frac{2}{\kappa} \cdot \frac{b}{2 b+1}+\frac{\varepsilon}{\kappa}$ for $\kappa / 2+1 \leq i \leq \kappa, w_{i}=0$ for $\kappa+1 \leq i \leq n$, and $\bar{q}=\frac{2}{\kappa} \cdot \frac{b^{2}+b}{2 b+1}$. With this, we have $[q ; w]=[\bar{q} ; \bar{w}],\|\bar{w}\|_{1}=1,\|w-\bar{w}\|_{1} \geq \frac{1}{2 b+1}-\varepsilon>\frac{1}{3} \cdot \frac{\Delta}{q}-\varepsilon$, and $\|w-\bar{w}\|_{\infty} \geq$ $\frac{1}{\kappa(2 b+1)}-\frac{\varepsilon}{\kappa}>\frac{\Delta}{3(\Delta+1)} \cdot \frac{\Delta}{q}-2 \Delta \varepsilon$. If instead $\kappa \equiv 1(\bmod 2)$, then we have $\kappa \geq 3$. In this case we set $\bar{w}_{i}=\frac{2}{\kappa} \cdot \frac{b+1}{2 b+1}-\frac{\varepsilon}{\kappa}$ for $1 \leq i \leq(\kappa-1) / 2, \bar{w}_{i}=\frac{2}{\kappa} \cdot \frac{b}{2 b+1}+\frac{\varepsilon}{\kappa}$ for $(\kappa+1) / 2 \leq i \leq \kappa-1, \bar{w}_{\kappa}=\frac{1}{\kappa}, w_{i}=0$ for $\kappa+1 \leq i \leq n$, and $\bar{q}=\frac{2}{\kappa} \cdot \frac{b^{2}+b}{2 b+1}$. With this, we have $[q ; w]=[\bar{q} ; \bar{w}],\|\bar{w}\|_{1}=1,\|w-\bar{w}\|_{1} \geq \frac{1}{2 b+1} \cdot\left(1-\frac{1}{\kappa}\right)-\varepsilon>\frac{2}{9} \cdot \frac{\Delta}{q}-\varepsilon$, and $\|w-\bar{w}\|_{\infty} \geq \frac{1}{\kappa(2 b+1)}-\frac{\varepsilon}{\kappa}>\frac{\Delta}{3(\Delta+1)} \cdot \frac{\Delta}{q}-2 \Delta \varepsilon$. 


\section{PROOF.(Lemma 3.14)}

If $\hat{q} \leq 1-\hat{q}$, we can apply Lemma A.1 and Lemma A.2 with $0<\varepsilon<\min \left\{\frac{1}{10}, \frac{\Delta}{45 \hat{q}}\right\}$. For the other case we remark that the dual of each weighted game $[q ; w]$ is given by $[1-q+\tilde{\varepsilon} ; w]$ for each $0<\tilde{\varepsilon}<\min \left\{w_{i} \mid i \in N, w_{i}>0\right\}$. So, we can apply Lemma A.1 and Lemma A.2 with $q=1-\hat{q}+\tilde{\varepsilon}$ and $0<\varepsilon<\frac{1}{2} \cdot \min \left\{\frac{1}{10}, \frac{\Delta}{45 q}\right\}=: \kappa$. Certainly, we can choose $\varepsilon>0$ small enough to get

$$
\left\|\min \left\{2, \frac{\Delta}{\min \{\hat{q}, 1-\hat{q}\}}\right\}-\min \left\{2, \frac{\Delta}{\min \{q, 1-q\}}\right\}\right\|_{1}<\kappa
$$

and $q \leq 1-q$. The stated result then follows from the triangle inequality.

\section{REFERENCES}

[1] N. Alon and P. H. Edelman. The inverse Banzhaf problem. Social Choice and Welfare, 34(3):371$377,2010$.

[2] Y. Bachrach, E. Markakis, E. Resnick, A. D. Procaccia, J. S. Rosenschein, and A. Saberi. Approximating power indices: theoretical and empirical analysis. Autonomous Agents and Multi-Agent Systems, 20(2):105-122, 2010.

[3] J. F. Banzhaf III. Weighted voting doesn't work: a mathematical analysis. Rutgers Law Review, 19:317, 1964.

[4] G. Chalkiadakis, E. Elkind, and M. Wooldridge. Computational aspects of cooperative game theory. Synthesis Lectures on Artificial Intelligence and Machine Learning, 5(6):1-168, 2011.

[5] P.-L. Chang, V. C. Chua, and M. Machover. LS Penrose's limit theorem: tests by simulation. Mathematical Social Sciences, 51(1):90-106, 2006.

[6] B. Curry and K. D. George. Industrial concentration: a survey. The Journal of Industrial Economics, 31(3):203-255, 1983.

[7] A. De, I. Diakonikolas, and R. A. Servedio. The inverse Shapley value problem. Games and Economic Behavior, 105:122-147, 2017.

[8] P. Dubey and L. S. Shapley. Mathematical properties of the Banzhaf power index. Mathematics of Operations Research, 4(2):99-131, 1979.

[9] S. S. Fatima, M. Wooldridge, and N. R. Jennings. A linear approximation method for the Shapley value. Artificial Intelligence, 172(14):1673-1699, 2008.

[10] J. Freixas and S. Kaniovski. The minimum sum representation as an index of voting power. European Journal of Operational Research, 233(3):739-748, 2014.

[11] J. Freixas and S. Kurz. On $\alpha$-roughly weighted games. International Journal of Game Theory, 43(3):659-692, 2014.

[12] Z. Galil. The nucleolus in games with major and minor players. International Journal of Game Theory, 3(3):129-140, 1974.

[13] T. Gvozdeva, L. A. Hemaspaandra, and A. Slinko. Three hierarchies of simple games parameterized by "resource" parameters. International Journal of Game Theory, 42(1):1-17, 2013.

[14] N. Houy and W. S. Zwicker. The geometry of voting power: weighted voting and hyper-ellipsoids. Games and Economic Behavior, 84:7-16, 2014.

[15] A. Jelnov and Y. Tauman. Voting power and proportional representation of voters. International Journal of Game Theory, 43(4):747-766, 2014.

[16] S. Kaniovski and S. Kurz. The average representation -a cornucopia of power indices? Homo Oeconomicus, 32(2):169-181, 2015. 
[17] S. Kaniovski and S. Kurz. Representation-compatible power indices. Annals of Operations Research, pages 1-31, to appear.

[18] V. M. Kartak, A. V. Ripatti, G. Scheithauer, and S. Kurz. Minimal proper non-irup instances of the one-dimensional cutting stock problem. Discrete Applied Mathematics, 187:120-129, 2015.

[19] Y. Koriyama, A. Macé, R. Treibich, and J.-F. Laslier. Optimal apportionment. Journal of Political Economy, 121(3):584-608, 2013

[20] S. Kurz. On minimum sum representations for weighted voting games. Annals of Operations Research, 196(1):361-369, 2012.

[21] S. Kurz. On the inverse power index problem. Optimization, 61(8):989-1011, 2012.

[22] S. Kurz. The inverse problem for power distributions in committees. Social Choice and Welfare, 47(1):65-88, 2016.

[23] S. Kurz, N. Maaser, and S. Napel. On the democratic weights of nations. Journal of Political Economy, 125(5):1599-1634, 2017.

[24] S. Kurz and S. Napel. Heuristic and exact solutions to the inverse power index problem for small voting bodies. Annals of Operations Research, 215(1):137-163, 2014.

[25] S. Kurz, S. Napel, and A. Nohn. The nucleolus of large majority games. Economics Letters, 123(2):139-143, 2014.

[26] M. Laakso and R. Taagapera. Effective number of parties: A measure with application to Western Europe. Comparative Political Studies, 12(1):3-27, 1979.

[27] M. Laakso and R. Taagepera. Proportional representation and effective number of parties in Finland. In Power, voting, and voting power, pages 107-120. Springer, 1982.

[28] D. Leech. Ownership concentration and the theory of the firm: a simple-game-theoretic approach. The Journal of Industrial Economics, pages 225-240, 1987.

[29] D. Leech. Computing power indices for large voting games. Management Science, 49(6):831-837, 2003.

[30] D. Leech. Power indices in large voting bodies. Public Choice, 155(1-2):61-79, 2013.

[31] D. Leech and J. Leahy. Ownership structure, control type classifications and the performance of large british companies. The Economic Journal, 101(409):1418-1437, 1991.

[32] I. Lindner and M. Machover. LS Penrose's limit theorem: proof of some special cases. Mathematical Social Sciences, 47(1):37-49, 2004.

[33] I. Lindner and G. Owen. Cases where the Penrose limit theorem does not hold. Mathematical Social Sciences, 53(3):232-238, 2007.

[34] A. Neyman. Renewal theory for sampling without replacement. The Annals of Probability, pages 464-481, 1982.

[35] B. Peleg. On weights of constant-sum majority games. SIAM Journal on Applied Mathematics, 16(3):527-532, 1968.

[36] L. S. Penrose. On the objective study of crowd behaviour. HK Lewis, 1952.

[37] D. Schmeidler. The nucleolus of a characteristic function game. SIAM Journal on Applied Mathematics, 17(6):1163-1170, 1969.

[38] L. S. Shapley and M. Shubik. A method for evaluating the distribution of power in a committee system. American Political Science Review, 48(3):787-792, 1954.

[39] J. von Von Neumann and O. Morgenstern. Theory of Games and Economic Behavior. Princeton University Press, 3rd edition, 1953.

SASCHA KURZ, UNIVERSity OF BAYREUTH, 95440 BAYREUTH, GERMANY

E-mail address: sascha.kurz@uni-bayreuth. de 